\title{
Kinase-independent inhibition of cyclophosphamide-induced pathways protects the ovarian reserve and prolongs fertility
}

\author{
Giovanna Bellusci', Luca Mattiello', Valentina lannizzotto ${ }^{1}$, Sarah Ciccone ${ }^{1}$, Emiliano Maiani ${ }^{1}$, Valentina Villani ${ }^{1}$, \\ Marc Diederich ${ }^{2}$ and Stefania Gonfloni ${ }^{1}$
}

\begin{abstract}
Premature ovarian failure and infertility are adverse effects of cancer therapies. The mechanism underlying chemotherapy-mediated depletion of the ovarian reserve remains unclear. Here, we aim to identify the signaling pathways involved in the loss of the ovarian reserve to prevent the damaging effects of chemotherapy. We evaluated the effects of cyclophosphamide, one of the most damaging chemotherapeutic drugs, against follicle reserve. In vivo studies showed that the cyclophosphamide-induced loss of ovarian reserve occurred through a sequential mechanism. Cyclophosphamide exposure induced the activation of both DNAPK- $\mathrm{H} 2 \mathrm{AX}$-checkpoint kinase 2 (CHK2)p53/TAp63a isoform and protein kinase B (AKT)-forkhead box O3 (FOXO3a) signaling axes in the nucleus of oocytes. Concomitant administration of an allosteric ABL inhibitor and cyclophosphamide modulated both pathways while protecting the ovarian reserve from chemotherapy assaults. As a consequence, the fertility of the treated mice was prolonged. On the contrary, the administration of an allosteric ABL activator enhanced the lethal effects of cyclophosphamide while shortening mouse fertility. Therefore, kinase-independent inhibition may serve as an effective ovarian-protective strategy in women under chemotherapy.
\end{abstract}

\section{Introduction}

Premature ovarian failure and infertility are frequent consequences of cancer therapy ${ }^{1}$. Chemotherapy triggers the degeneration of the ovarian reserve $e^{2,3}$, a source of fertilizable eggs during the entire reproductive life of a female. Preserving the ovarian reserve during cancer treatment is a major concern for the maintenance of fertility and to ameliorate the quality of life of survivors. Studies in transgenic mouse models have revealed the role of several molecules in the maintenance of ovarian reserve following ionization radiation (IR) treatment. The alpha

\footnotetext{
Correspondence: Stefania Gonfloni (stefania.gonfloni@uniroma2.it)

${ }^{1}$ Department of Biology, University of Rome Tor Vergata, via della Ricerca Scientifica, 00133 Rome, Italy

${ }^{2}$ College of Pharmacy, Seoul National University, 1 Gwanak-ro, Gwanak-gu, Seoul 08826, Republic of Korea

These authors contributed equally: Giovanna Bellusci, Luca Mattiello, Valentina lannizzotto, Sarah Ciccone
}

Edited by I. Amelio
TAp63 isoform (TAp63 $\alpha$ ) was thought to be a key mediator of IR-induced ovarian reserve loss ${ }^{4}$. Lack of TAp63 $\alpha$ expression in mouse oocytes promoted resistance to the lethal effects of $\mathrm{IR}^{5}$. IR-induced TAp63 activation depended on a conformational transition of the molecule $^{6}$. The pro-apoptotic proteins PUMA and NOXA may act as downstream effectors of TAp63 $\alpha^{7}$. In mice, checkpoint kinase 2 (CHK2) is essential for DNA damage surveillance in female meiosis ${ }^{8}$. Moreover, the lack of CHK2 expression affects TAp63 activation following IR treatment ${ }^{8}$. In vitro experiments in ovarian culture system showed that the transient administration of a CHK2 inhibitor II hydrate may preserve oocyte viability following IR treatment ${ }^{9}$. Another study demonstrated that CHK2 inhibitor II hydrate affected the activities of a broad spectrum of kinases and impacted the global repression of the DNA damage response (DDR) in cultured ovaries ${ }^{10}$, thereby raising some questions about its specificity. Tuppi

\section{(c) The Author(s) 2019}

(c) (i) Open Access This article is licensed under a Creative Commons Attribution 4.0 International License, which permits use, sharing, adaptation, distribution and reproduction cc) in any medium or format, as long as you give appropriate credit to the original author(s) and the source, provide a link to the Creative Commons license, and indicate if changes were made. The images or other third party material in this article are included in the article's Creative Commons license, unless indicated otherwise in a credit line to the material. If material is not included in the article's Creative Commons license and your intended use is not permitted by statutory regulation or exceeds the permitted use, you will need to obtain permission directly from the copyright holder. To view a copy of this license, visit http://creativecommons.org/licenses/by/4.0/. 


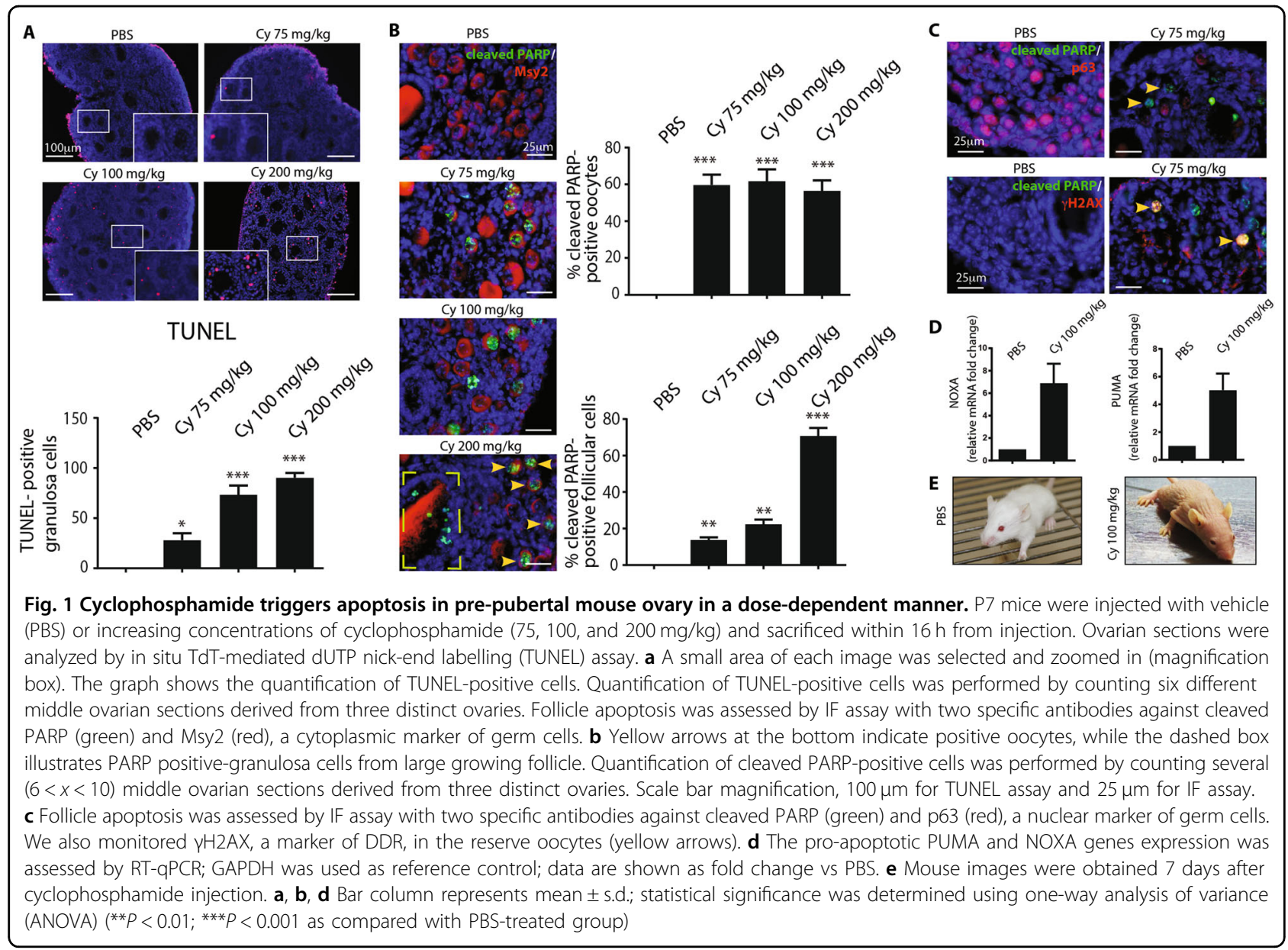

et al. reported that CHK2 and the executioner kinase CK1 are both involved in the TAp63 $\alpha$-mediated oocyte degeneration in cultured ovaries exposed to chemotherapy $^{11}$. As cultured mouse ovaries exposed to chemotherapeutic drugs may not recapitulate the signaling pathways physiologically occurring in the ovary in vivo, we aimed to perform all our experiments under in vivo conditions. We evaluated the effects of cyclophosphamide, a chemotherapeutic agent commonly used for the treatment of pediatric cancer patients. Cyclophosphamide is transformed in the liver into active alkylating metabolites, which induce the formation of DNA adducts. How cyclophosphamide contributes to ovarian follicle depletion is yet incompletely understood and is debatable $^{12}$. Meirow et al. suggest that the mechanism underlying cyclophosphamide-induced oocyte loss comprised the accelerated activation of primordial follicle that results in a "burnout" effect ${ }^{13}$. However, chemotherapeutic drugs may directly damage the primordial follicle and induce apoptosis. The extent of ovarian reserve loss also depends on the type and dosage of the chemotherapeutic agent ${ }^{14}$. Understanding the molecular basis underlying the effect of chemotherapy on quiescent primordial follicles is therefore essential for the identification of an effective cotreatment that may simultaneously preserve fertility in women $^{15,16}$. Here, we investigated the in vivo consequences of different doses of cyclophosphamide on the ovarian reserve. We used a pre-pubertal mouse model to evaluate the effect of transient administration of small molecule kinase inhibitors to identify putative fertoprotective adjuvants ${ }^{17}$ to limit the damaging effects of chemotherapy.

\section{Results}

\section{Cyclophosphamide triggers a dose-dependent loss of primordial follicles}

We investigated the effects of cyclophosphamide on perinatal mouse ovaries. We injected mice (at postnatal day 7, P7) with different doses of cyclophosphamide (ranging from 50 to $200 \mathrm{mg} / \mathrm{kg}$ ). One day after injection, ovaries were dissected and analyzed with TdT-mediated dUTP nick-end labelling (TUNEL), immunofluorescence (IF), and immunohistochemistry (IHC) assays. Cyclophosphamide administration induced a dose-dependent increase in the number of TUNEL-positive granulosa cells surrounding the growing follicles (Fig. 1a). Cell death in 
the ovarian follicle reserve was observed with IF staining for cleaved poly ADP ribose polymerase (PARP) (Fig. 1b). We found apoptotic oocytes in the groups treated with low doses of cyclophosphamide $(50-75 \mathrm{mg} / \mathrm{kg})$, while higher doses of cyclophosphamide $(100-200 \mathrm{mg} / \mathrm{kg})$ activated cleaved PARP in the granulosa cells of large growing follicles (see dashed yellow box in Fig. 1b). We also detected the phosphorylation of the histone variant $\mathrm{H} 2 \mathrm{AX}$ at Ser139 residue, also named as $\gamma \mathrm{H} 2 \mathrm{AX}$, an early hallmark of DDR, in the nucleus of cyclophosphamidedamaged oocytes (Fig. 1c). We also detected the enhanced transcription of PUMA and NOXA genes by RT-qPCR (Fig. 1d). Temporary hair loss was reported a week later in cyclophosphamide-injected animals (Fig. 1e).

\section{DNAPK is activated in the nucleus of reserve oocytes following cyclophosphamide treatment}

To investigate the mechanism underlying cyclophosphamide-induced follicle death, we evaluated the activation of the apical DDR kinase (DNAPK) with phospho-specific antibodies after $16 \mathrm{~h}$ of cyclophosphamide injection. The presence of apoptosis in the primordial/primary follicle population was consistent with the activation of DNAPK in the nucleus of reserve oocytes (Fig. 2a). Phosphorylation of the histone variant $\mathrm{H} 2 \mathrm{AX}$ at Ser139 was observed in the nucleus of damaged oocytes. The majority of oocytes were positive for both DNAPK and $\gamma \mathrm{H} 2 \mathrm{AX}$ expression at every dose of cyclophosphamide (Fig. 2a). Confocal images of middle ovarian sections obtained from cyclophosphamide-injected mice showed that the oocytes with a strong signal for $\gamma \mathrm{H} 2 \mathrm{AX}$ or p-DNAPK had lower expression of TAp63 (Fig. 2b). Western blot analysis of the dissected ovaries revealed the cyclophosphamide-induced phosphorylation of both $\gamma \mathrm{H} 2 \mathrm{AX}$ and $\mathrm{p} 53$. In addition, cyclophosphamide-induced TAp63 activation, as indicated by a mobility shift (*) that was absent in the control group (Fig. 2c).

We observed activation of checkpoint kinase CHK2 and p53 in the nucleus of the damaged oocytes (Supplementary Fig. 1A). Both CHK2 and p53 are involved in the removal of oocytes with unrepaired meiotic DNA doublestrand breaks $(\mathrm{DBSs})^{8}$. Confocal images show phosphorylated p53 and concomitant lower expression of TAp63 in the oocyte nucleus (Supplementary Fig. 1B). ChIP assay performed on cyclophosphamide-injected ovaries shows that p53 activation led to transcription of proapoptotic gene PUMA (Supplementary Fig. 1C). Thus, the cyclophosphamide-induced loss of ovarian reserve involves the activation of the DNAPK- $\gamma \mathrm{H} 2 \mathrm{AX}-\mathrm{CHK} 2-$ p53/TAp63 $\alpha$ signaling axes.

We investigated the mechanisms underlying the cyclophosphamide-induced follicle death following the activation of the protein kinase B (AKT) pathway using phospho-specific antibodies after $16 \mathrm{~h}$ treatment with cyclophosphamide (Supplementary Fig. 2). The AKT pathway is involved in primordial follicle activation through the regulation of the activity of the forkhead box O3 (FOXO3a) transcription factor ${ }^{18}$. Upon phosphorylation, AKT may enter the nucleus and phosphorylate FOXO3a, which eventually leaves the nucleus and enables follicular activation.

Following cyclophosphamide injection, p-AKT and pFOXO3a were detected by confocal microscopy (Supplementary Fig. 2B) in the nucleus of reserve oocytes and in a dose-dependent manner (Supplementary Fig 2A, upper and lower panel). We also observed concomitant expression of p-AKT and $\gamma \mathrm{H} 2 \mathrm{AX}$ (Supplementary Fig. 2A, central panel) and p-AKT/pFOXO3a with p-ATM (Supplementary Fig. 2C). Thus, the presence of the early marker of DDR after cyclophosphamide treatment was consistent with the activation of the AKT-FOXO3a pathway.

\section{GNF-2 prevents $\mathrm{YH} 2 \mathrm{AX}$ phosphorylation in the nucleus of reserve oocytes}

Previous studies have revealed the protective role of an ABL kinase inhibitor (imatinib) on ovarian reserve in cisplatin-induced degeneration ${ }^{19-22}$. However, the mechanism underlying oocyte protection by imatinib remains unclear. As discussed in a recent paper ${ }^{10}$, cisplatin induced a response that activated the pathways different from those activated by IR. Cisplatin toxicity gradually accumulated from multiple sources (lipids, proteins, etc.) and induced integrated pathways of the two p53 homologs (TAp63 $\alpha$ and TAp73 $\alpha)^{10}$ in follicles.

Here, we tested whether the allosteric ABL compounds affect the DDR induced by cyclophosphamide. In our experiments, we used an allosteric activator of $\mathrm{ABL}$ kinase $^{23}$ (Supplementary Fig. 3) to validate the effect of $\mathrm{ABL}$ compounds in vivo. We treated female mice with cyclophosphamide in the presence of GNF-2. Concomitant administration of GNF-2 resulted in a significant reduction in the number of TUNEL-positive granulosa cells in a dose-dependent manner (Fig. 3a). We failed to observe any apoptosis in reserve oocytes (Fig. 3b). Cotreatment of cyclophosphamide and GNF-2 also affected DNAPK activation and the consequent $\gamma \mathrm{H} 2 \mathrm{AX}$ phosphorylation in the nucleus of reserve oocytes (Fig. 3d). Also, the transcription of proapoptotic genes PUMA and NOXA is affected by GNF-2 (Fig. 3c).

On the contrary, the concomitant administration of DPH (an allosteric ABL activator) enhanced DDR activation (Fig. 4) and oocyte apoptosis induced by cyclophosphamide.

IF assay results revealed the increase in the number of $\gamma \mathrm{H} 2 \mathrm{AX}$-positive oocytes (Fig. 4a), p-DNAPK positive oocytes (Fig. 4b) and cleaved PARP positive oocytes (Fig. 4c) after cyclophosphamide treatment. Therefore, we 


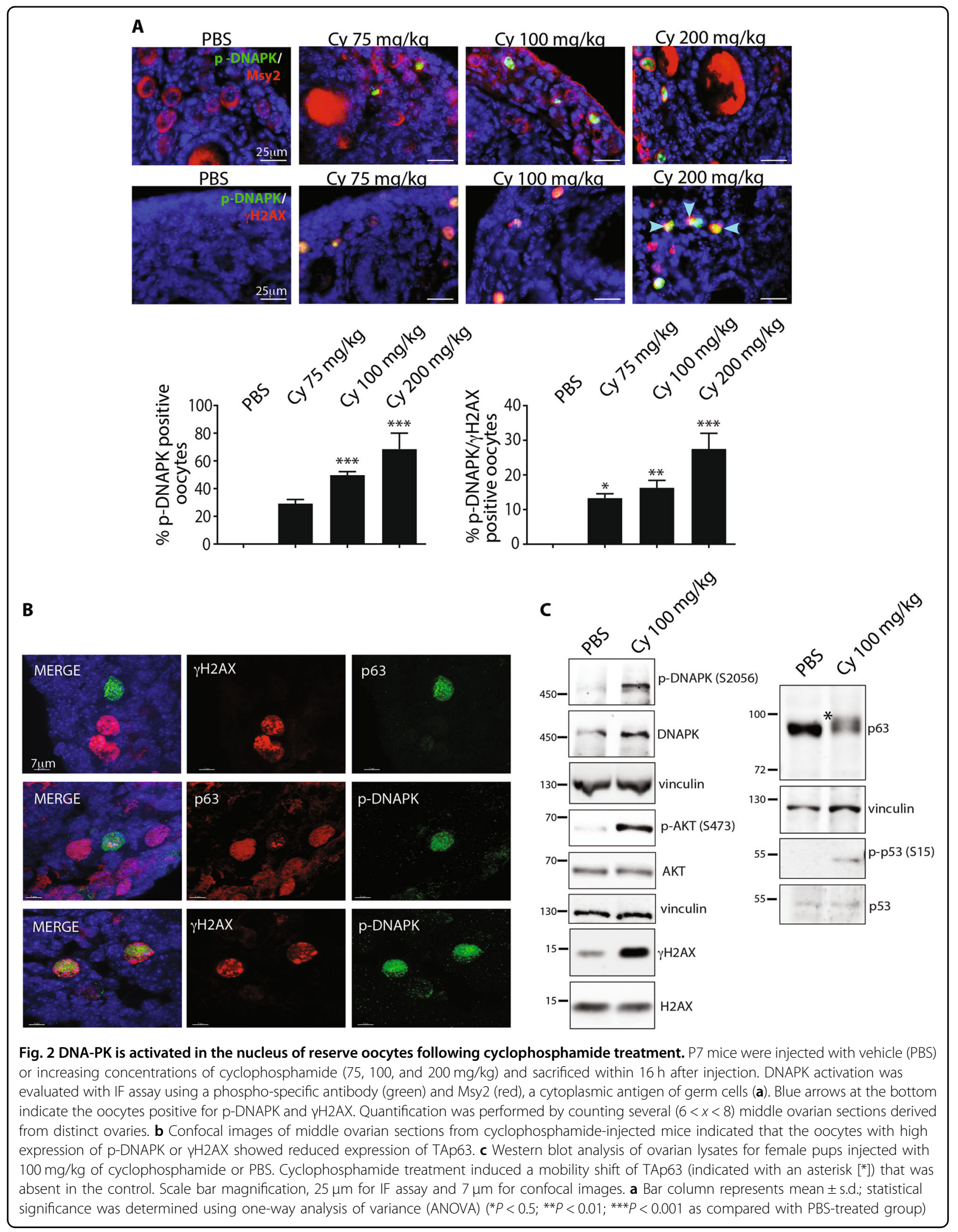




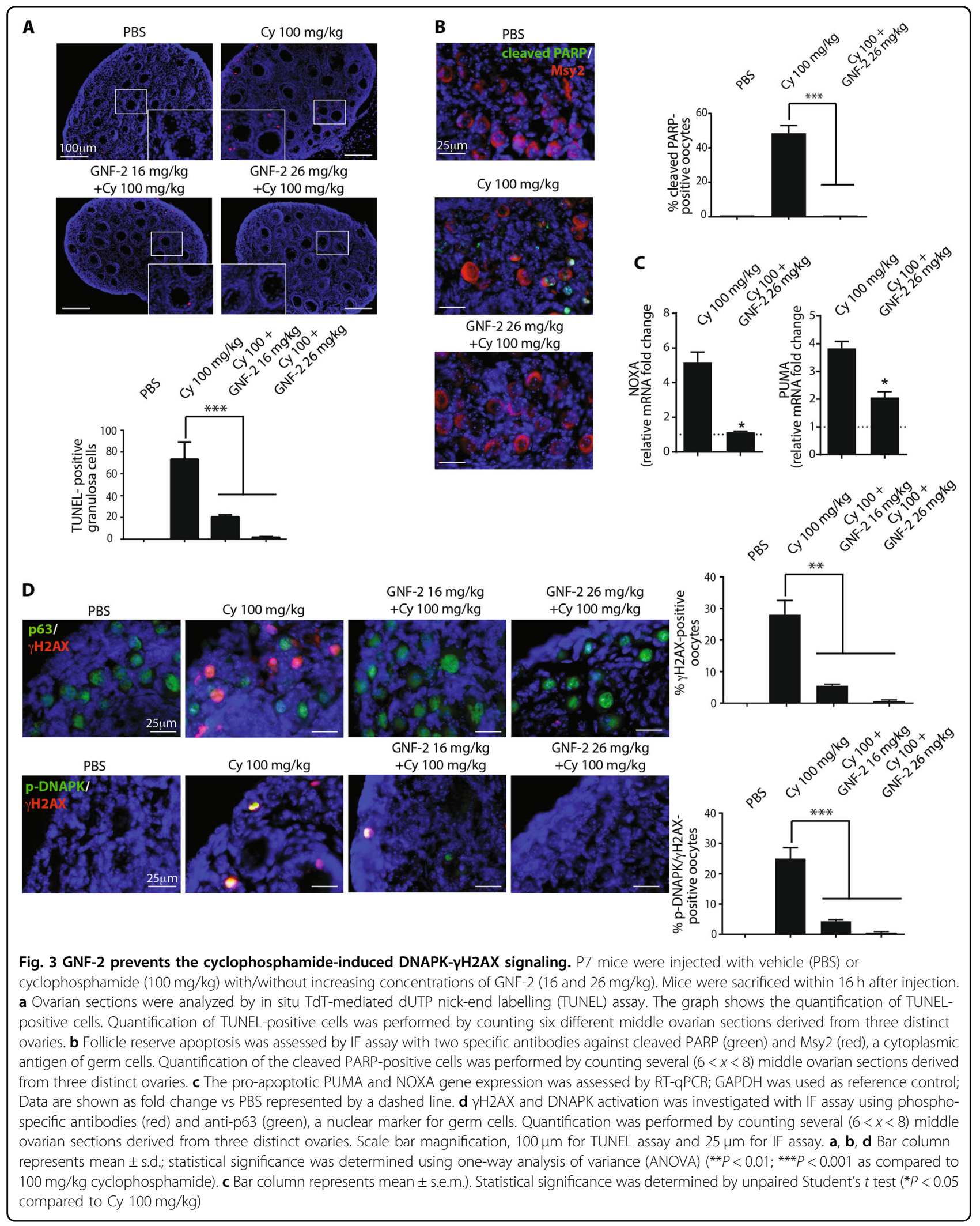




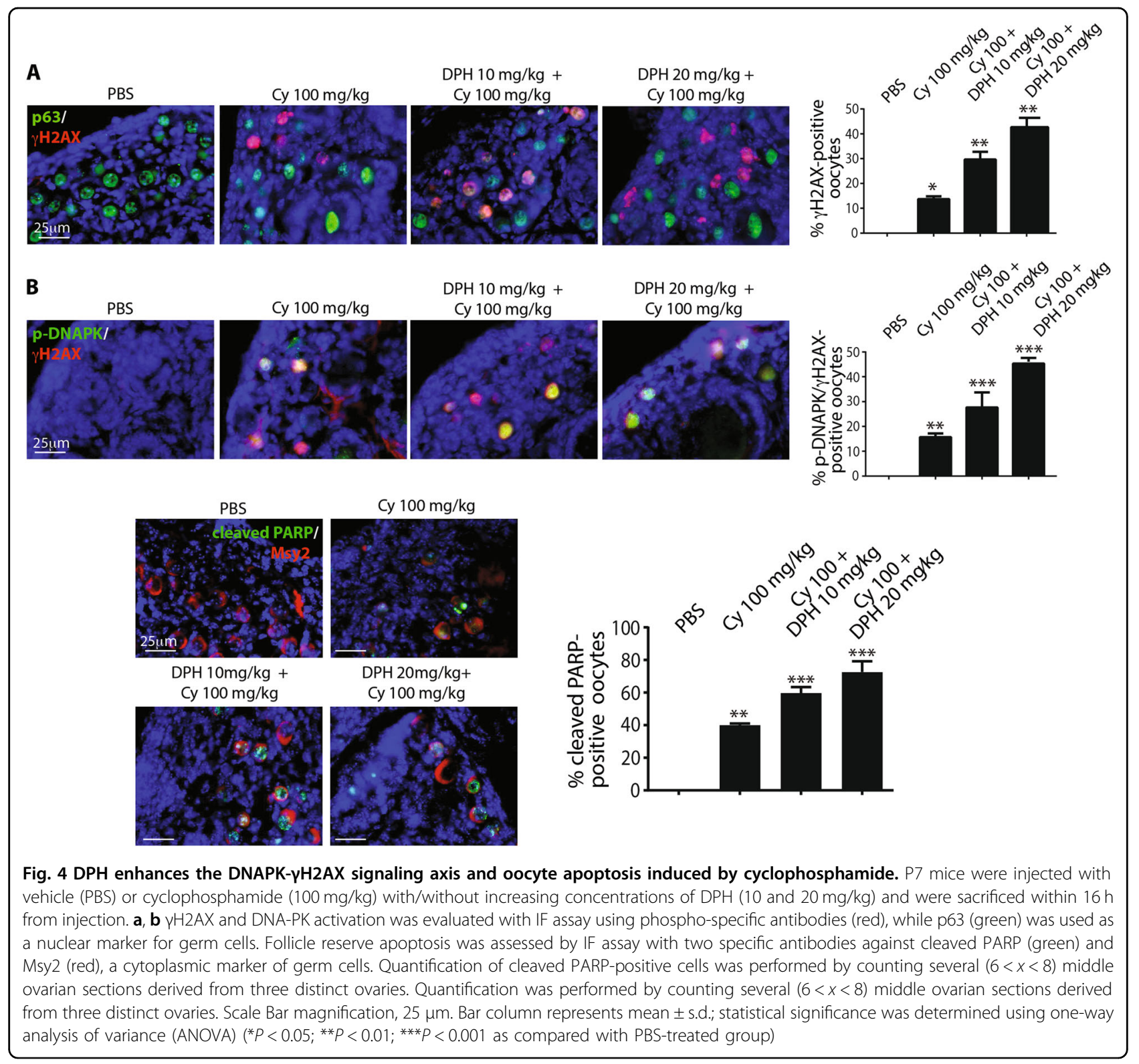

investigated whether ATM phosphorylates $\mathrm{H} 2 \mathrm{AX}$ in the damaged oocytes. IF assay results showed that ATM phosphorylation was coupled with a decrease in TAp63 expression in the nucleus of reserve oocytes, as reported for p-DNAPK (Supplementary Fig. 4). The concomitant administration of GNF-2 also prevented ATM activation in the nucleus of reserve oocytes.

In conclusion, GNF-2 and cyclophosphamide cotreatment restricted apoptosis in ovarian reserve through the prevention of the activation of the DNAPK(ATM)$\gamma \mathrm{H} 2 \mathrm{AX}-\mathrm{CHK} 2-\mathrm{p} 53 / \mathrm{Tap} 63$ signaling axes. We also evaluated the effect of allosteric compounds on the activation of the AKT-FOXO3a pathway. Co-treatment of cyclophosphamide with GNF-2 prevented the translocation of
p-AKT in the nucleus of the oocyte (Supplementary Fig. 5A), while DPH enhanced the activation of AKT and concomitant phosphorylation of FOXO3a (Supplementary Fig. 5D, E) in the ovarian reserve assaulted by cyclophosphamide.

\section{GNF-2 prevents the cyclophosphamide-induced loss of follicle reserve}

We dissected and analyzed the cyclophosphamidetreated ovaries by western blotting. Ovarian lysates from mice injected with PBS or cyclophosphamide $(100 \mathrm{mg} / \mathrm{kg})$ alone or in combination with kinase inhibitors targeting ABL (GNF-2, imatinib), DNAPK (NU7441), or ATM (KU55933) revealed the GNF-2-mediated prevention of 


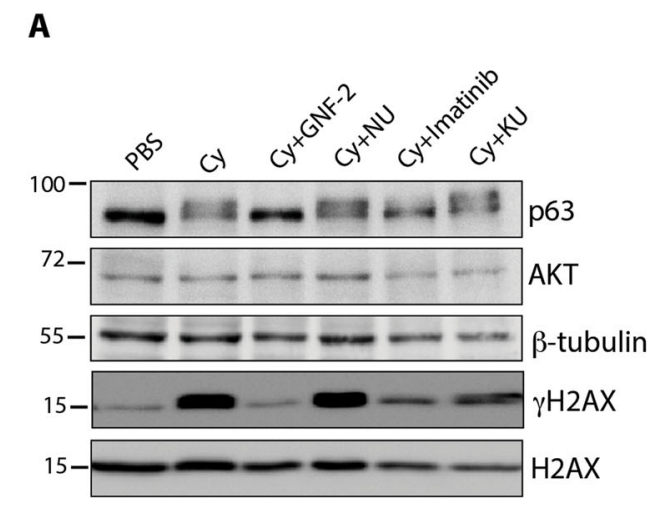

B

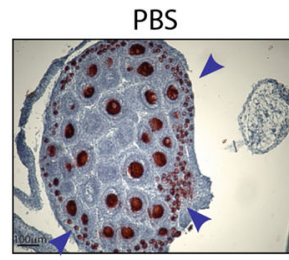

$\mathrm{NU} 3,5 \mathrm{mg} / \mathrm{kg}+$ Cy $100 \mathrm{mg} / \mathrm{kg}$

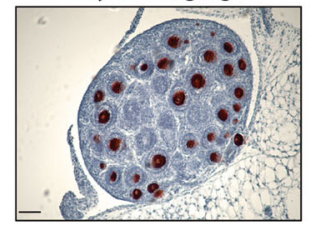

GNF-2 $26 \mathrm{mg} / \mathrm{kg}+$

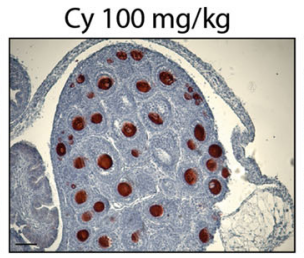

Imatinib $12 \mathrm{mg} / \mathrm{kg}+$ Cy $100 \mathrm{mg} / \mathrm{kg}$

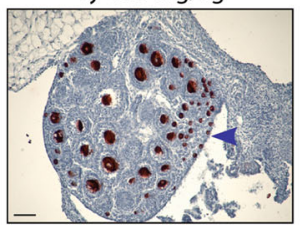

Cy $100 \mathrm{mg} / \mathrm{kg}$

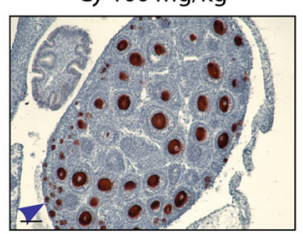

$\mathrm{KU} 10 \mathrm{mg} / \mathrm{kg}+$ Cy $100 \mathrm{mg} / \mathrm{kg}$

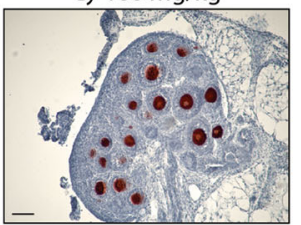

C

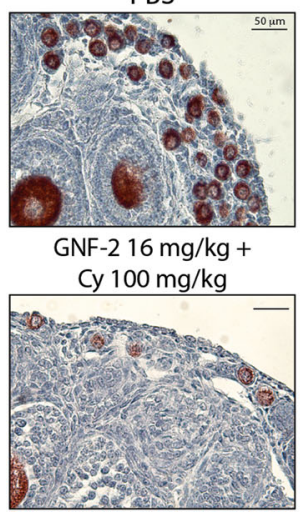

Cy $100 \mathrm{mg} / \mathrm{kg}$

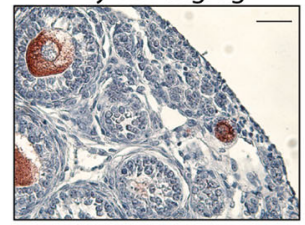

GNF-2 $26 \mathrm{mg} / \mathrm{kg}+$ Cy $100 \mathrm{mg} / \mathrm{kg}$

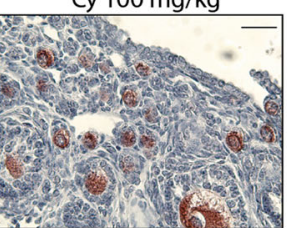

D

primordial/primary follicles

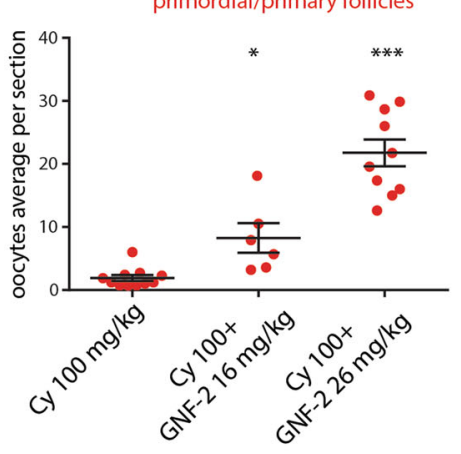

secondary follicles

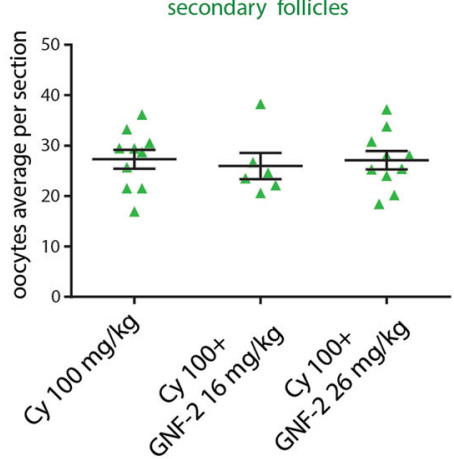

Fig. 5 GNF-2 prevents cyclophosphamide-induced loss of reserve follicles. P7 mice were injected with vehicle (PBS) or cyclophosphamide $(100 \mathrm{mg} / \mathrm{kg}$ ) alone or in combination with different kinase inhibitors (GNF-2, imatinib, NU7441, or KU55933). Mice were sacrificed within $24 \mathrm{~h}$ from injection. a Western blot analysis showed that GNF-2 prevented TAp63 activation/shift and YH2AX phosphorylation, whereas NU7441 and KU55933 were less effective in preventing TAp63 activation/shift. b, c Ovaries were dissected 3 days after injection and analyzed with IHC assay using Msy-2 antibody. $\mathbf{d}$ Several ovaries from three independent experiments were analyzed; each dot in the box plot represents the average number (primordial/ primary or secondary) of follicles per section of each gonad collected. Scale Bar magnification, $100 \mu \mathrm{m}$ (b), $50 \mu \mathrm{m}$ (c). Statistical significance was determined using one-way analysis of variance (ANOVA) ${ }^{*} P<0.05 ;{ }^{* *} P<0.001$ as compared with the group treated with $100 \mathrm{mg} / \mathrm{kg}$ cyclophosphamide). Blue arrows indicate follicle reserves

TAp63 activation (i.e., absence of mobility shift) and $\gamma \mathrm{H} 2 \mathrm{AX}$ generation. NU7441 and KU55933 did not prevent TAp63 activation or $\gamma \mathrm{H} 2 \mathrm{AX}$ generation (Fig. 5a). On the other hand, imatinib partially prevented TAp63 activation/shift and $\gamma \mathrm{H} 2 \mathrm{AX}$ expression. These results were also confirmed by IHC assay performed on the ovaries dissected 3 days after injection (Fig. 5b). Ovarian sections showed a massive depletion in primordial and primary follicles after cyclophosphamide treatment, whereas a significant rescue in follicle reserve was observed in the ovaries co-treated with cyclophosphamide and GNF-2 (Fig. 5c). We counted primordial, primary, and secondary follicles from middle-ovary sections (Fig. 5d), as previously described ${ }^{22}$. We failed to observe any significant difference in secondary follicles in cyclophosphamidetreated and control groups.
Imatinib and NU7441 partially mitigate the toxic effect of cyclophosphamide

We evaluated the effect of different doses of two kinase inhibitors imatinib and NU7441. Both compounds bind to the ATP-binding cleft of kinases and are quite selective for their main target kinases. However, imatinib binds to other kinases, such as platelet-derived growth factor receptor (PDGFR) and c-KIT ${ }^{24}$, while NU7441 also inhibits phosphatidylinositol-4,5-bisphosphate 3-kinase (PI3K) and has little activity against ATM and $\mathrm{ATR}^{25}$. We assessed the effect of these compounds in combination with cyclophosphamide. We injected mice with cyclophosphamide $(100 \mathrm{mg} / \mathrm{kg})$ in the presence of each inhibitor. TUNEL assay result showed that the co-treatment with imatinib or NU7441 had milder effects on the prevention of granulosa cell death than co-treatment with 


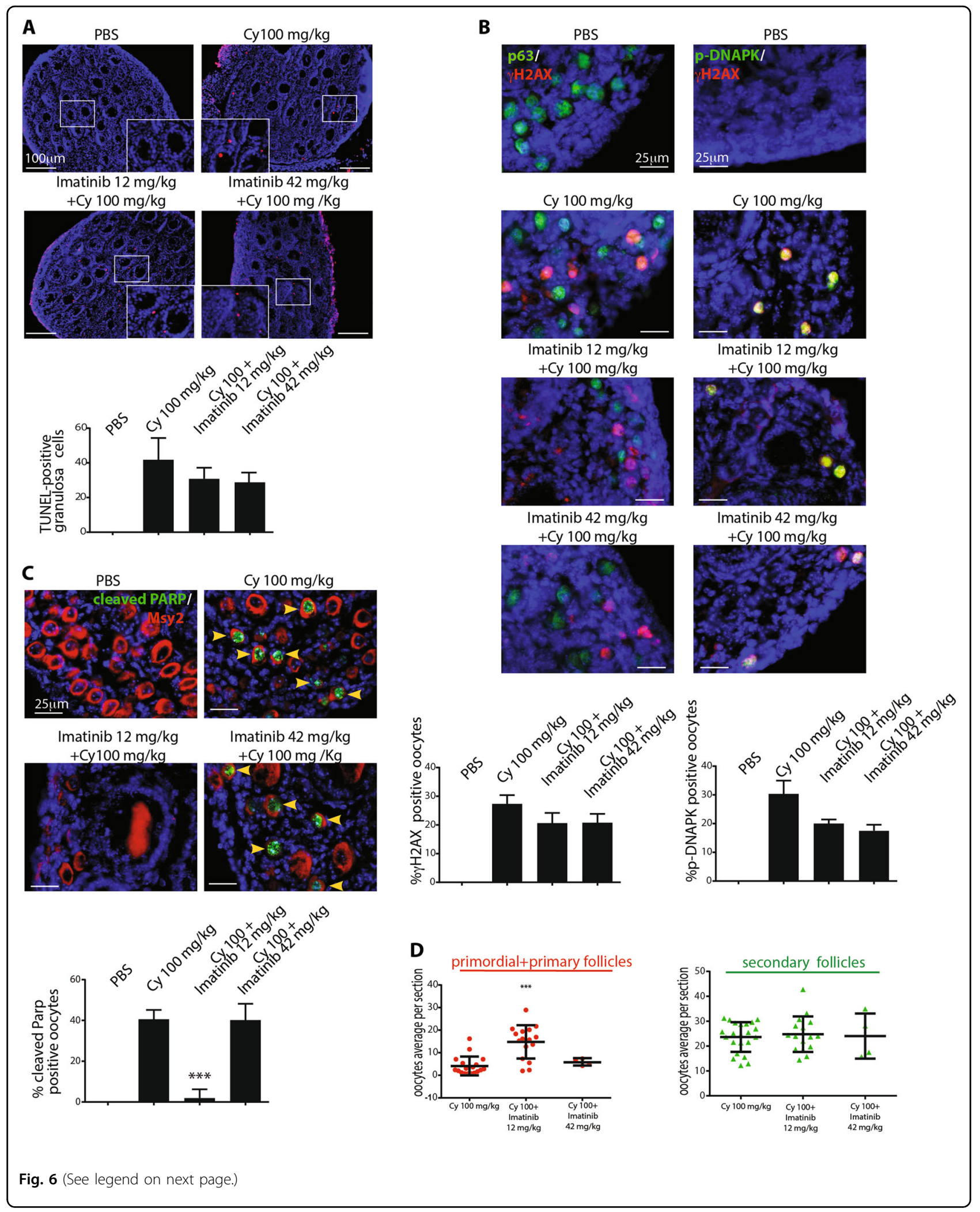


(see figure on previous page)

Fig. 6 Imatinib partially prevents oocyte apoptosis induced by cyclophosphamide. P7 mice were injected with vehicle (PBS) or

cyclophosphamide $(100 \mathrm{mg} / \mathrm{kg}$ ) with/without increasing concentrations of imatinib (12 and $42 \mathrm{mg} / \mathrm{kg}$ ) and sacrificed within $16 \mathrm{~h}$ from injection. a Ovarian sections were analyzed by in situ TdT-mediated dUTP nick-end labelling (TUNEL) assay. The graph shows the quantification of TUNELpositive cells. Quantification of TUNEL-positive cells was performed by counting six different middle ovarian sections derived from three distinct ovaries. b $\mathrm{YH} 2 \mathrm{AX}$ and DNAPK activation was observed with IF assay using phospho-specific antibodies and p63 was used as a nuclear marker for germ cells. Quantification was performed by counting several $(6<x<8)$ middle ovarian sections derived from three distinct ovaries. Co-staining for $p$ DNAPK and $\mathrm{YH} 2 \mathrm{AX}$ showed the activation of DNA damage response in reserve oocytes. Quantification was performed by counting several $(6<x<8)$ middle ovarian sections derived from three distinct ovaries. c Ovarian reserve apoptosis was assessed by IF assay using antibodies against cleaved PARP (green) and Msy2 (red), a cytoplasmic antigen of germ cells. Quantification of cleaved PARP-positive cells was performed by counting several $(6<x<8)$ middle ovarian sections derived from three distinct ovaries. $\mathbf{a}-\mathbf{c}$ Bar column represents mean \pm s.d.; statistical significance was determined using one-way analysis of variance (ANOVA) ( ${ }^{* *} P<0.001$ as compared with the group treated with $100 \mathrm{mg} / \mathrm{kg}$ cyclophosphamide). d Ovaries dissected 3 days after injection were analyzed with IHC assay using Msy2 antibody (Fig. 5b). Ovaries from three independent experiments were analyzed; each dot in the box plot represents the average number of follicles (primordial + primary and secondary) per section of each gonad collected. Statistical significance was determined using one-way analysis of variance (ANOVA) ${ }^{* * *} P<0.001$ as compared with cyclophosphamidetreated group at $100 \mathrm{mg} / \mathrm{kg}$ ). Scale bar magnification, $100 \mu \mathrm{m}$ for TUNEL assay and $25 \mu \mathrm{m}$ for IF assay

GNF-2 (Fig. 6a and Supplementary Fig. 6A). We found that imatinib and NU7441 failed to prevent DNAPK$\gamma$ H2AX-TAp63 activation (Fig. $6 \mathrm{~b}$ and Supplementary Fig. 6B). However, at low dose, imatinib temporarily prevented apoptosis in follicle reserve (Fig. 6c). This observation was also confirmed by IHC assay (Fig. 5b) performed in ovarian sections dissected after 3 days of injection (Fig. 6d). NU7441 and cyclophosphamide cotreatment mildly mitigated apoptosis in follicle reserve (Supplementary Fig. 6C) as confirmed by IHC staining (Fig. 5b) of the ovarian sections dissected 3 days after injection (Supplementary Fig. 6D). In conclusion, imatinib and NU7441 are less effective than the allosteric inhibitor GNF-2 in the prevention of cyclophosphamide-induced follicle loss. This observation may depend on the inhibition of kinases, including the receptor tyrosine kinase cKIT and PIK3 that play important roles in follicle reserve. On the contrary, no known target for the allosteric compound GNF-2, other than ABL kinases, has been reported so $\mathrm{far}^{26}$.

\section{GNF-2 prolongs fertility in female mice injected with cyclophosphamide}

To investigate the long-term effect of concomitant GNF-2 administration, co-treated mice were allowed to grow and eventually mated with fertile males. Mice were injected at P7, 3 days after few ovaries from each experimental group were analyzed with $\mathrm{IHC}$ assay (Fig. 7a and Supplementary Fig. 7A). We also monitored hair recovery for each experimental group after 2 weeks of injection (Supplementary Fig. 7B) and evaluated pubertal ovaries of cyclophosphamide-treated mice by IHC staining before fertility test (Supplementary Fig. 8). In addition, we measured the average weight of mice from each experimental group over 4 weeks from injection (Fig. 7b). We followed the mating capability and the number of pups delivered during six breeding rounds. No evident differences in behavior and development were observed between the off-springs from each experimental group during the first post-natal week. However, we observed that fertility was impaired in the females injected with cyclophosphamide and DPH (Fig. 7c, d). The mice treated with cyclophosphamide and DPH were infertile after three mating rounds, quite before those treated with cyclophosphamide. On the other hand, co-treatment of mice with GNF-2 and cyclophosphamide prolonged the fertility as compared to cyclophosphamide-treated mice and increased the cumulative number of pups compared to cyclophosphamide + DPH and cyclophosphamide groups (Fig. 7e). The gross morphology of the ovaries dissected from adult mice showed a clear difference in size between gonads of each experimental group. The analysis of ovarian sections by IHC assay with Msy2 confirmed the nearly complete absence of follicle reserve in the ovaries from cyclophosphamide + DPH and cyclophosphamide groups (Fig. 7f).

\section{Discussion}

Cyclophosphamide is currently used for the treatment of pediatric cancers. A major concern is the ovarian reserve depletion induced by cyclophosphamide treatment. In the present study, we used a pre-pubertal mouse model to show in vivo the signaling axes induced by cyclophosphamide. We also tested small molecules that could limit the cyclophosphamide-induced toxicity by acting as putative "ferto-adjuvants".

Genetically modified mouse models have facilitated the study of the signaling pathways induced in ovary by IR or chemotherapy. Gene targeting approaches may oversimplify the interpretation of the results. The lack of a single node/protein could affect signaling circuits at different levels and the observations reported here in knockout mice may be attributed to the multiple effects that are difficult to dissect. Small molecules offer unique advantages in terms of interpretation of results because of their transient inhibitory effects on targeted proteins 


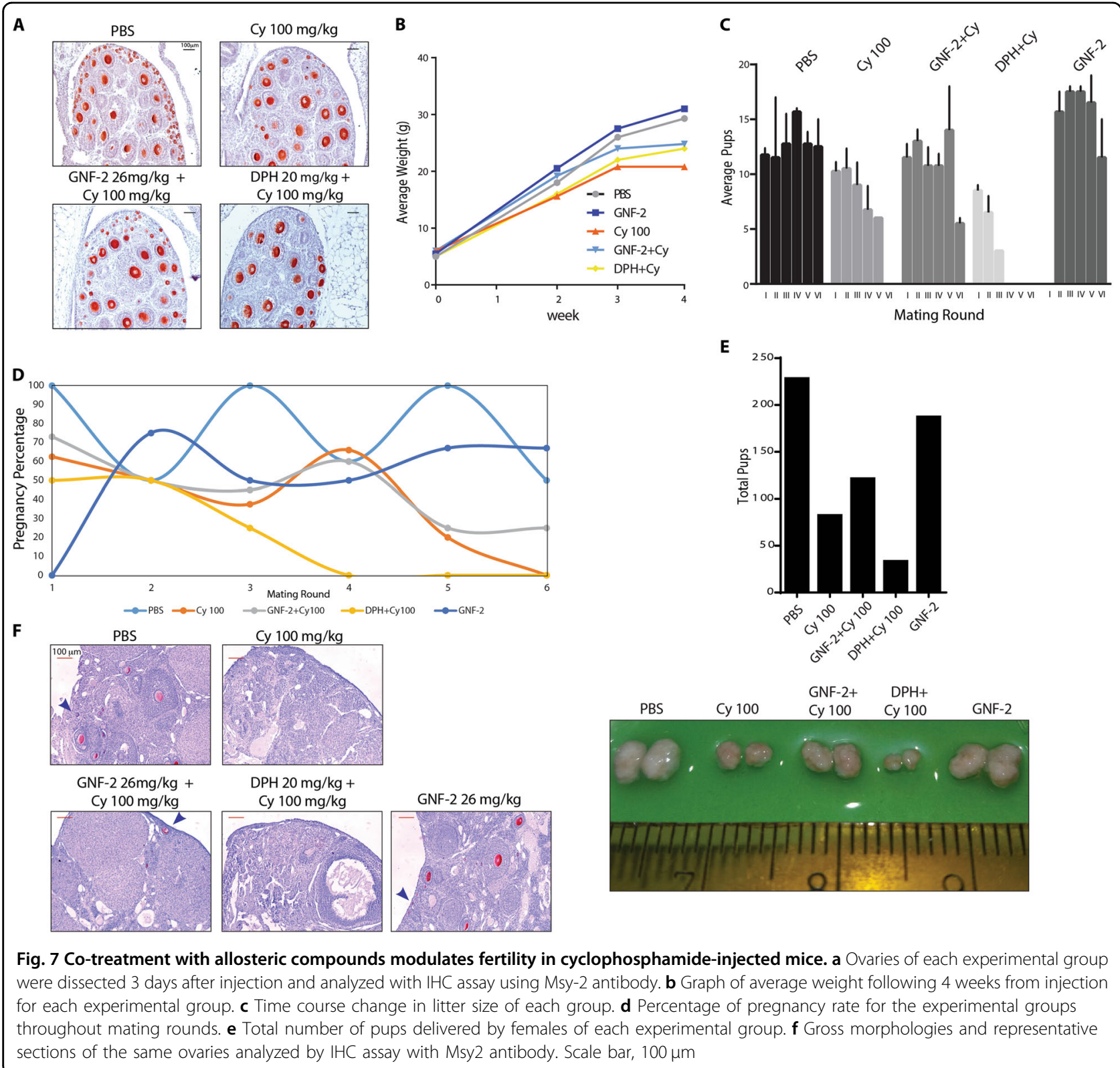

(and/or pathways). Pharmaceutical inhibitors may have a direct relevance with patient care.

Our study showed that cyclophosphamide-induced apoptosis in the ovarian reserve through signaling axes involving DNAPK/(ATM), CHK2, p53, and TAp63 $\alpha$. In addition, the co-treatment of cyclophosphamide and GNF-2 affected the DDR induced by cyclophosphamide in the ovary. We also showed that the reserve oocytes rescued from immediate degeneration were healthy enough to produce normal off-springs. Gross morphology and IHC analyses of ovary sections performed either before fertility test or after infertility detection in the first group of treated mice confirmed that the concomitant administration of GNF-2 and cyclophosphamide had long-term effects. Our data are reinforced through the use of an allosteric activator (DPH) that exerted opposite effects as compared to GNF-2. Co-treatment with DPH and cyclophosphamide enhanced the DDR induced by cyclophosphamide in ovaries and shortened mouse fertility. We also compared the effect of the transient administration of inhibitors targeting DDR apical kinases and observed mild protective effects exclusively at low doses of kinase inhibitors. Why allosteric compounds targeting ABL are more effective than ATP-competitive kinase inhibitors in rewiring apoptotic pathways induce by cyclophosphamide is questionable. We hypothesized that either (i) the theory of "burnout effect"13 or (ii) the direct DNA damage of follicle reserve in the ovary assaulted by 


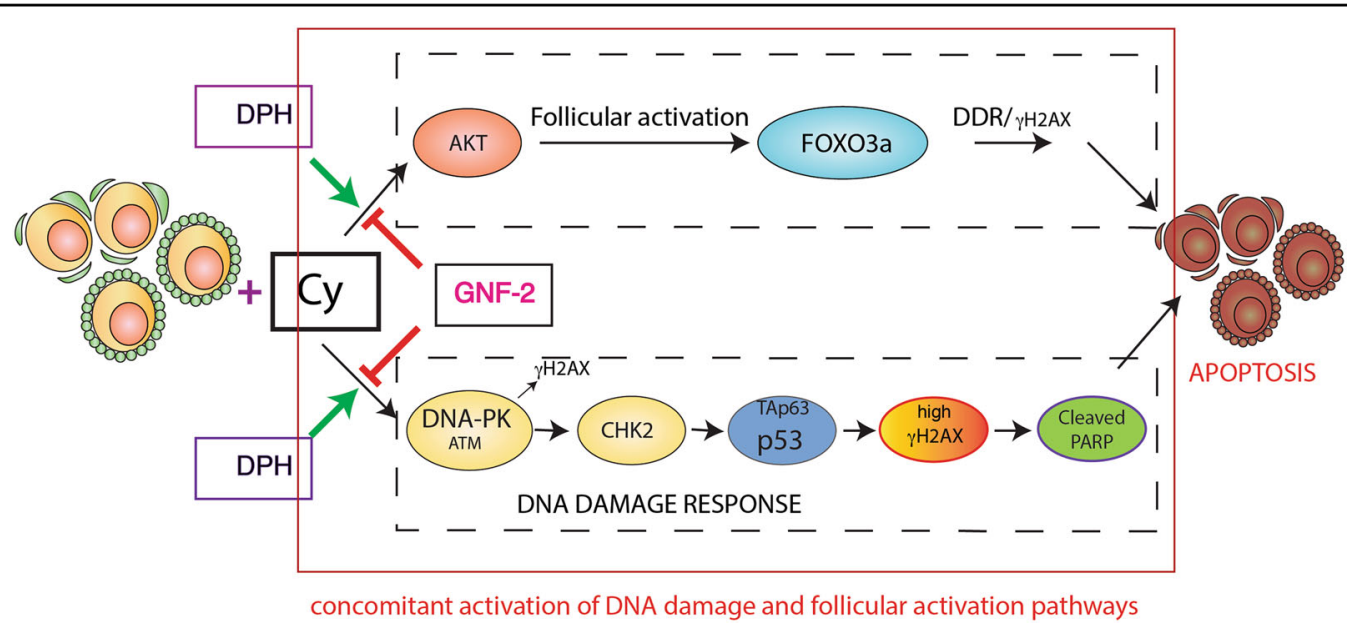

Fig. 8 Proposal model. Concomitant administration of GNF-2 and cyclophosphamide results in the inhibition of the AKT-FOXO3a signaling axis and DDR, thereby preventing cyclophosphamide-induced apoptosis of oocyte reserve. On the contrary, DPH enhanced the activation of the AKT-FOXO3a signaling axis and DDR response following cyclophosphamide injection. Oocyte reserve was positive for both p-AKT/p-FOXO3a and p-ATM/YH2AX; thus, the presence of the early marker of DDR was consistent with the activation of the AKT-FOXO3 pathway. This observation suggests that oocytes leave their follicular quiescence to initiate the DNA damage signaling pathway that leads to oocyte apoptosis

cyclophosphamide may be associated with this effect. Both theories rely on the fact that the damaged somatic cells communicate with the oocyte. In this scenario, allosteric ABL compounds could affect follicle apoptosis by targeting the transmission of stress signals from pregranulosa cells to the oocyte. Although the molecular details remain unclear, our current model suggests that ABL kinases may act as "allosteric devices" that contribute to fuel the molecular events underlying stress signaling/ DDR in the follicles ${ }^{27}$. This model is supported by the observation that GNF-2 prevents the activation of the AKT-FOXO3 signaling axis induced by cyclophosphamide. We also found that DPH promotes the activation of the AKT-FOXO3a signaling axis following cyclophosphamide treatment and that the reserve oocytes were positive for both p-AKT/p-FOXO3a and p-ATM/ $\gamma \mathrm{H} 2 \mathrm{AX}$ expression. Thus, the presence of the early marker of DDR is consistent with the activation of the AKTFOXO3a pathway. These observations suggest that oocytes leave their follicular quiescence to initiate the DNA damage signaling pathways that end in oocyte apoptosis (Fig. 8). Whether this is a general mechanism occurring with other DNA-damaging chemotherapeutic drugs and IR is yet unknown.

Evidences suggest that ABL kinase inhibitors protect the ovarian reserve from cisplatin-induced degeneration ${ }^{19-22}$. However, the mechanism underlying the imatinibmediated protection of oocytes remains debated. Genetic studies have shown that the oocyte ABL kinases are dispensable for cisplatin-induced TAp63 $\alpha$ activation ${ }^{10}$. However, ABL kinases in complex with inhibitors may not recapitulate the results observed in ABL null mice, given that ABL proteins take part to opposing signaling pathways in cells ${ }^{28}$.

Recent studies have identified CHK2 and p53 as two important players in the efficient removal of oocytes with unrepaired meiotic DNA $\mathrm{DBSs}^{8}$. In addition, CHK2dependent TAp63 $\alpha$ phosphorylation is a key event induced in response to $\mathrm{IR}^{6,8}$. We show that cyclophosphamide-induced DNA damage checkpoint pathways that involve signaling of DNAPK to CHK2, which in turn communicates with p53 and TAp63, revising a previously proposed model $^{8}$. We found p-p53 (Ser15) in the nucleus of the damaged oocytes, supporting the direct role of p53 in driving the apoptotic response of follicles. This observation is intriguing, as TAp63 $\alpha$ is considered as a key player in quality control of germ cells. However, activated TAp63 $\alpha$ exerts modest activity as a transcription factor as compared with $\mathrm{p} 53$, as we confirmed by ChIP experiments (Supplementary Fig. 1). Thus, the quality control and apoptotic response induced by cyclophosphamide require the predominant activity of $\mathrm{p} 53$.

A recent work showed that mouse models lacking PUMA (a pro-apoptotic protein) retained fertility after chemotherapy $^{29}$. This observation supports the results that reserve oocytes from PUMA ${ }^{-1-}$ mice may sufficiently repair themselves to support healthy off-springs. These findings strengthen the argument that oocytes are in fact capable of efficient DNA repair in response to the inhibition of the apoptotic pathway in the ovary ${ }^{30}$.

The concomitant administration of GNF-2 and cyclophosphamide results in the inhibition of the AKTFOXO3a signaling axis and DDR, thereby preventing 
cyclophosphamide-induced apoptosis of ovarian reserve. Thus, the feasibility of ferto-adjuvant therapies based on allosteric ABL compounds is established. With this in mind, we should consider that the systemic administration of allosteric inhibitors may interfere with the efficacy of cancer therapies. Clinical applications may warrant studies to improve the targeted delivery of these compounds to the gonads. Despite these efforts, the discovery of Asciminib (ABL001) $)^{31,32}$, the first allosteric ABL inhibitor used in clinic, offers promises to develop fertoprotective strategies suitable for pediatric cancer patients.

\section{Materials and methods}

\section{Animals and injection}

All procedures involving mice and care have been conducted at the Interdepartmental Service Centre- Station for Animal Technology (STA), University of Rome "Tor Vergata", in accordance with the ethical standards, according to the Declaration of Helsinki, in compliance with our institutional animal care guidelines and following national and international directives (Italian Legislative Decree 26/2014, Directive 2010/62/EU of the European Parliament and of the Council). The ovaries were collected from CD-1 mice (Charles River) of 6 to 8 days old. Newborn mice (P6) were treated with intraperitoneal (ip) injection with $\mathrm{PBS}$ or cyclophosphamide (50, 75, 100, and $200 \mathrm{mg}$ per $\mathrm{kg}$ of body weight). Mice were pre-treated with different inhibitors for $1 \mathrm{~h}$ before cyclophosphamide injection using a sterile micro syringe (Becton Dickson). Inhibitors used: GNF2 (range $15 \mathrm{mg}-26 \mathrm{mg}$ per $\mathrm{kg}$ of body weight), IMATINIB (12-42 mg per $\mathrm{kg}$ of body weight), NU (2-5 mg per $\mathrm{kg}$ of body weight), KU (10 mg per $\mathrm{kg}$ of body weight). Cyclophosphamide (BAXTER) was prepared fresh as concentrated $40 \mathrm{mg} / \mathrm{ml}$ in PBS. We dissolved Imatinib methane sulfonate salt (Novartis) in water, GNF-2 (SIGMA), NU (TOCRIS) and KU55993 (TOCRIS) in DMSO.

\section{Immunohistochemistry, Follicle counting, and statistical analysis}

We prepared sections from ovaries fixed in MetaCarnoy solution (as previously described (Gonfloni et al 2009), embedded in paraffin and cut in slices of $5-7 \mu \mathrm{m}$ of thickness. Sections were dewaxed, re-hydrated, and microwaved. Slices were then permeabilized with PBS triton $0,2 \%$ and incubated with MSY-2 antibody (Santa Cruz). The staining was performed with immunocruz staining system for anti-goat antibody (Santa Cruz, sc2023) and 3-aminoethyl-9-ethylcarbazole as substrate (AEC, Sigma). Sections were counterstained with hematoxylin and cover-slipped with Aquatex. Quantification of primordial and primary or secondary follicles was derived from histological analysis, counting Msy2-positive germ cells of mid-ovary sections. For each ovary (P9), several central slices $(10<n<15)$ are included in the counting, with the exception of smaller peripheral slices (12-14 on average per each ovary). Quantification of primordial/ primary follicle reserve is expressed as mean of immature follicles (primordial plus primary follicles) per single ovary. Average values for each ovary are represented as discrete points on a scatter plot. Mean value \pm S.D. are shown in the scatter plot. The analysis of variance is evaluated with one-way ANOVA, with Turkey multiple comparison Test using PRISM 6 (Graph Pad software) $\left({ }^{*} P<0.05 ;{ }^{* *} P<0.01 ;{ }^{* * *} P<0.001\right)$ or by unpaired Student's $t$ test where indicated.

\section{Immunofluorescence}

We prepared sections from ovaries sections fixed in MetaCarnoy solution, embedded in paraffin and cut in slice of 5-7 $\mu \mathrm{m}$ of thickness. Sections were dewaxed rehydrated and microwaved in sodium citrate $10 \mathrm{mM}$ pH6, to expose the antigens. Unspecific-binding sites were blocked by incubating sections for $2 \mathrm{~h}$ in a blocking solution (PBS plus 1\%glycine, 5\% BSA, 5\% FBS and 5\% NGS (normal goat serum). Ovaries sections were then incubated overnight with antibodies against MSY-2, p63, p-ATM, $\gamma \mathrm{H} 2 \mathrm{AX}, \mathrm{p}-\mathrm{DNA}-\mathrm{PK}, \mathrm{p}-\mathrm{p} 53$, p-CHK2, p-DNAPK, pAKT, p-FOXO3a and cleaved PARP. After washing in PBS triton $0,05 \%$, tissue sections were incubated with Alexa 555-goat anti-mouse (life technologies) and alexa 488-goat anti rabbit (invitrogen).

\section{Immunoblot analysis}

P7 dry ice-frozen ovaries were homogenized with a mini-pestle in ice-cold lysis buffer $(50 \mathrm{mM}$ Tris- $\mathrm{HCl} \mathrm{pH}$ 7.5, $150 \mathrm{mM} \mathrm{NaCl}, 0.5 \% \mathrm{NP}-40,5 \mathrm{mM}$ EDTA, $0.5 \%$ sodium deoxycholate, $1 \mathrm{mM}$ phenylmethylsulfonyl fluoride, $1 \mathrm{mM}$ sodium o-vanadate, $10 \mu \mathrm{g} \mathrm{ml}^{-1}$ Tosyl phenylalanyl chloromethyl ketone (TPCK), $10 \mu \mathrm{g} \mathrm{ml}^{-1}$, Tosyl-L-lysyl-chloromethane hydrochloride (TLCK) supplemented with protease inhibitors, all purchased from SIGMA). Equal amounts of protein extract (equivalent of one up to three ovaries) was loaded onto $6 \%, 8 \%$ or $12 \%$ SDS-PAGE gel and transferred to a nitrocellulose membrane (Amersham Bioscience).

\section{Tunel}

Ovary sections were stained according to the Fluorescein In Situ Cell Death detection Kit (Roche Diagnostic) and analyzed with a fluorescent filter. We used the protocol recommended by the manufacturer. DAPI (Molecular Probes Inc.)

\section{RNA isolation an RT-qPCR}

P7 dry ice-frozen ovaries were homogenized with a mini-pestle in TRIzol Reagent (Thermo Fisher Scientific), and RNA was extracted according to manufacturer's instructions. To generate cDNA for RT-qPCR, total RNA 
was solubilized in ribonuclease-free water and used for reverse transcription by PrimeScript ${ }^{\mathrm{Tm}}$ RT Reagent Kit (Perfect Real Time) (Takara). Primers used were obtained from Sigma-Aldrich and are as follows:

\begin{tabular}{lll} 
Gene & Forward & Reverse \\
Puma & AGGGAAGGGAGGGCTGAAGG & GAGGCCAGGCCCAAAGTGAA \\
Noxa & CGCTGGTGCTGCCTACTGAA & GCCTITCTCCCGGGCATCTC \\
GAPDH & AAGGGCTCATGACCACAGTC & CAGGGATGATGTTCTGGGCA \\
\hline
\end{tabular}

Real-time qPCR was performed using the $\mathrm{SYBR}^{\circledast}$ Premix Ex Taq (Tli RNase H Plus) (Takara) on a StepOne real-time PCR System (Applied Biosystems). All reactions were run as triplicates. Data were analyzed by the StepOne $^{\text {tw }}$ Software (v2.3) using the second-derivative maximum method. The fold changes in mRNA levels were relative to a control after normalization to GAPDH.

\section{Chromatin Immunoprecipitation (ChIP)}

Ovaries collected were cross-linked for $15 \mathrm{~min}$ at room temperature with $1 \%$ formaldehyde. The reaction was stopped by $5 \mathrm{~min}$ incubation in $125 \mathrm{mM}$ glycine. Chromatin was sonicated using Branson Sonifier, seven cycles for $20 \mathrm{~s}$ ON, $20 \mathrm{~s}$ OFF. Average size of sonicated DNA was around $500-1200 \mathrm{bp}$, as measured by agarose gel electrophoresis. Samples were pre-cleared with pre-adsorbed Salmon Sperm A-coupled Sepharose beads, and overnight immunoprecipitated with anti -P63 (CST 13109) or antip53 (DO-1 sc-126) antibodies. Normal mouse or rabbit IgG were used as controls. Precipitated chromatin complexes were eluted by $150 \mu \mathrm{l}$ of TE-SDS buffer (1\% SDS, $10 \mathrm{mM}$ Tris- $\mathrm{HCl}, 5 \mathrm{mM}$ EDTA) for $15 \mathrm{~min}$. Finally, the protein-DNA cross-links were reversed by overnight incubation at $65^{\circ} \mathrm{C}$. DNA amplification was performed using SYBR ${ }^{\circledast}$ Premix Ex Taq (Tli RNase H Plus) (Takara) on a StepOne real-time PCR System (Applied Biosystems). All reactions were run as triplicates. Data were analyzed by the StepOne ${ }^{\text {tw }}$ Software (v2.3) using the second- derivative maximum method. Results are expressed as fold enrichment respect to IgG control. Primers used are as follows:

\begin{tabular}{lll} 
Gene & Forward & Reverse \\
Puma Response Element & CCTCTGGCTGCCGGG & CCGCCCCGCCTCTCGC \\
& AAACCCCCC & TGGCTCC \\
\hline
\end{tabular}

\section{Reagents}

Antibodies Msy-2 (sc-21316), Abl K-12 (sc-131), $\beta$-tubulin (sc-9104), p53 DO-1 (sc-126) and p-AKT (T308) (sc-16646-R) were purchased from Santa Cruz; antibody for p-H2AX ( $\gamma \mathrm{H} 2 \mathrm{AX})(05-636), \mathrm{H} 2 \mathrm{AX}(07-627)$ and p-Tyr (4G10) (05-321) were purchased from Millipore; antibody for p-DNA-PK (S2056) (SAB4504169) and P63 (Y4A3) (P3362) were purchased from SIGMA, antibody for p-ATM (S1981) (200-301-500) was purchased from Rockland, polyclonal antibody for p63 was an homemade rabbit serum; antibody for p-P53 (S15) (9284), pCHK2 (T68) (2197), p-FOXO3a (S253) (9466), p-CHK2 (T68) (2197), P63 (CST 13109) and cleaved PARP (9544) were purchased from Cell Signaling Technology. Antibody for Abl (8E9) was purchased from BD-pharmingen. Secondary antibodies were purchased from Jackson Immunoresearch. All the antibodies were diluted in a blocking solution containing 5\% BSA in PBS tween 0,05\% for Western Blotting analysis and in a blocking solution containing $1 \%$ glycine, 5\% FBS, 5\% BSA and 5\% NGS for immunofluorescence.

\section{Mating protocol}

We injected five cohorts of newborn CD1 mice (25 total female pups) with a single dose of cyclophosphamide (100 mg per $\mathrm{kg}$ of body weight), GNF-2 (26 mg per $\mathrm{kg}$ body weight) and with cyclophosphamide in combination with GNF2 or DPH (20 mg per kg body weight). Injection with PBS was used as a control. Five-six weeks after injection, we mated the control and treated mice with proven fertile males at regular time intervals every 5-6 weeks. Once mating was established by the formation of the fertilization plug (this occurs within a week), we separated the females and allowed the pregnancies to progress until delivery. We kept the mice for a week with their pups and then separated them. After a week (without breast-feeding), we mated them again with proven fertile males.

\section{Acknowledgements}

We thank Gianni Cesareni and Luisa Castagnoli for their valuable support. We are indebted to Stefano Cannata, Stefano Pirrò, Fabio Ciccarone and Sergio Bernardini for their technical advice. We are grateful to Gianni Cesareni and Luca Jovine for their helpful comments on the paper. This work was supported by grants from AIRC (IG11344) to S.G.

\section{Author contributions \\ G.B. performed the majority of the experiments with the help of V.I. L.M. performed IF assays for p-CHK2 and p-53, RT-qPCR, ChIP assay and the fertility test. S.C. performed confocal analysis and contributed to follicle quantification. E.M. performed all experiments shown in Supplementary Fig. 4. V.V. performed IHC assay with adult ovaries and follicle quantification shown in \\ Supplementary Fig. 8. M.D. provided support and reagents and helped with the critical reading of the paper. S.G. designed and directed the study, wrote the paper}

\section{Conflict of interest}

The authors declare that they have no conflict of interest.

\section{Publisher's note}

Springer Nature remains neutral with regard to jurisdictional claims in published maps and institutional affiliations. 
Supplementary Information accompanies this paper at (https://doi.org/ 10.1038/s41419-019-1961-y).

Received: 4 March 2019 Revised: 7 August 2019 Accepted: 27 August 2019 Published online: 27 September 2019

\section{References}

1. Morgan, S., Anderson, R. A., Gourley, C., Wallace, W. H. \& Spears, N. How do chemotherapeutic agents damage the ovary? Hum. Reprod. Update $\mathbf{1 8}$ 525-535 (2012)

2. Oktem, O. \& Oktay, K. Quantitative assessment of the impact of chemotherapy on ovarian follicle reserve and stromal function. Cancer 110, 2222-2229 (2007).

3. Meirow, D., Biederman, H., Anderson, R. A. \& Wallace, W. H. Toxicity of chemotherapy and radiation on female reproduction. Clin. Obstet. Gynecol. $\mathbf{5 3}$ 727-739 (2010)

4. Suh, E. K. et al. p63 protects the female germ line during meiotic arrest. Nature 444, 624-628 (2006)

5. Livera, G. et al. p63 null mutation protects mouse oocytes from radio-induced apoptosis. Reproduction 135, 3-12 (2008).

6. Deutsch, G. B. et al. DNA damage in oocytes induces a switch of the quality control factor TAp63alpha from dimer to tetramer. Cell 144, 566-576 (2011).

7. Kerr, J. B. et al. DNA damage-induced primordial follicle oocyte apoptosis and loss of fertility require TAp63-mediated induction of Puma and Noxa. Mol. cell 48, 343-352 (2012).

8. Bolcun-Filas, E., Rinaldi, V. D., White, M. E. \& Schimenti, J. C. Reversal of female infertility by Chk2 ablation reveals the oocyte DNA damage checkpoint pathway. Science 343, 533-536 (2014).

9. Rinaldi, V. D., Hsieh, K., Munroe, R., Bolcun-Filas, E. \& Schimenti, J. C. Pharmacological inhibition of the DNA damage checkpoint prevents radiationinduced oocyte death. Genetics 206, 1823-1828 (2017).

10. Kim, S. Y. et al. Transient inhibition of p53 homologs protects ovarian function from two distinct apoptotic pathways triggered by anticancer therapies. Cell Death Differ 26, 502-515 (2018)

11. Tuppi, M. et al. Oocyte DNA damage quality control requires consecutive interplay of CHK2 and CK1 to activate p63. Nat. Struct. Mol. Biol. 25, 261-269 (2018).

12. Bedoschi, G., Navarro, P. A. \& Oktay, K. Chemotherapy-induced damage to ovary: mechanisms and clinical impact. Future Oncol. 12, 2333-2344 (2016).

13. Kalich-Philosoph, L. et al. Cyclophosphamide triggers follicle activation and "Burnout"; AS101 prevents follicle loss and preserves fertility. Sci. Transl. Med 5, 185ra162 (2013)

14. Jayasinghe, Y. L., Wallace, W. H. B. \& Anderson, R. A. Ovarian function, fertility and reproductive lifespan in cancer patients. Expert Rev. Endocrinol. Metab. 13, 125-136 (2018)
15. Kim, S. Y., Kim, S. K., Lee, J. R. \& Woodruff, T. K. Toward precision medicine for preserving fertility in cancer patients: existing and emerging fertility preservation options for women. J. Gynecol. Oncol. 27, e22 (2016).

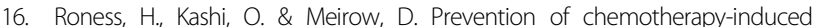
ovarian damage. Fertil. Steril. 105, 20-29 (2016).

17. Woodruff, T. K. Preserving fertility during cancer treatment. Nat. Med $\mathbf{1 5}$ 1124-1125 (2009)

18. Shah, J. S. et al. Biomechanics and mechanical signaling in the ovary: a systematic review. J. Assist Reprod. Genet 35, 1135-1148 (2018).

19. Gonfloni, S. et al. Inhibition of the c-Abl-TAp63 pathway protects mouse oocytes from chemotherapy-induced death. Nat. Med. 15, 1179-1185 (2009).

20. Kim, S. Y. et al. Rescue of platinum-damaged oocytes from programmed cell death through inactivation of the p53 family signaling network. Cell Death Differ. 20, 987-997 (2013).

21. Morgan, S., Lopes, F., Gourley, C., Anderson, R. A. \& Spears, N. Cisplatin and doxorubicin induce distinct mechanisms of ovarian follicle loss; imatinib provides selective protection only against cisplatin. PLOS ONE 8, e70117 (2013).

22. Maiani, E. et al. Reply to: cisplatin-induced primordial follicle oocyte killing and loss of fertility are not prevented by imatinib. Nat. Med 18, 1172-1174 (2012).

23. Yang, J. et al. Discovery and characterization of a cell-permeable, smallmolecule c-Abl kinase activator that binds to the myristoyl binding site. Chem. Biol. 18, 177-186 (2011)

24. Buchdunger, E. et al. Abl protein-tyrosine kinase inhibitor STI571 inhibits in vitro signal transduction mediated by c-kit and platelet-derived growth factor receptors. J. Pharm. Exp. Ther. 295, 139-145 (2000).

25. Tavecchio, M., Munck, J. M., Cano, C., Newell, D. R. \& Curtin, N. J. Further characterisation of the cellular activity of the DNA-PK inhibitor, NU7441, reveals potential cross-talk with homologous recombination. Cancer Chemother. Pharm. 69, 155-164 (2012).

26. Adrian, F. J. et al. Allosteric inhibitors of Bcr-abl-dependent cell proliferation Nat. Chem. Biol. 2, 95-102 (2006).

27. Gonfloni, S. Defying c-Abl signaling circuits through small allosteric compounds. Front Genet 5, 392 (2014).

28. Maiani, E., Diederich, M. \& Gonfloni, S. DNA damage response: the emerging role of c-Abl as a regulatory switch? Biochemical Pharmacol. 82, 1269-1276 (2011).

29. Nguyen, Q. N. et al. Loss of PUMA protects the ovarian reserve during DNAdamaging chemotherapy and preserves fertility. Cell Death Dis. 9, 618 (2018).

30. Stringer, J. M., Winship, A., Liew, S. H. \& Hutt, K. The capacity of oocytes for DNA repair. Cell Mol. Life Sci. https://doi.org/10.1007/s00018-018-2833-9 (2018).

31. Wylie, A. A. et al. The allosteric inhibitor ABL001 enables dual targeting of BCRABL1. Nature 543, 733-737 (2017).

32. Schoepfer, J. et al. Discovery of asciminib (ABL001), an allosteric inhibitor of the tyrosine kinase activity of BCR-ABL1. J. Med. Chem. 61, 8120-8135 (2018). 